\title{
Utilisation des drones pour la caractérisation des phénomènes d'érosion éolienne des dunes du littoral Atlantique. Exemple des Pays de Monts
}

\author{
Franck DESMAZES ${ }^{1}$, Manuel GARCIN ${ }^{1}$, Valentin METEREAU ${ }^{2}$, \\ Loïc GOUGUET ${ }^{2}$, Alexandre NICOLAE LERMA ${ }^{3}$, Jérome LEROUX ${ }^{4}$
}

1. BRGM, Direction Risques et Prévention, 3 Avenue Claude Guillemin, 4500 Orléans f.desmazes@brgm.fr;m.garcin@brgm.fr

2. ONF, Agence Pays de la Loire, 15 boulevard Léon Bureau, 44262 Nantes cedex 2 valentin.metereau@onf.fr ; loic.gouguet@onf.fr

3. BRGM, Direction des Actions Territoriales, Parc Technologique Europarc, 24 Avenue Léonard de Vinci, 33600 Pessac.

a.nicolaelerma@brgm.fr

4. Azur Drones, 98 avenue Général Leclerc, 92100 Boulogne Billancourt

jerome.leroux@azurdrones.com

\section{Résumé :}

Sur la côte Atlantique, l'ONF est gestionnaire de très vastes zones de dunes domaniales (380 km linéaire de massifs dunaires) et surveille l'érosion éolienne et anthropique de ce milieu sensible. Dans le cadre du suivi quinquennal de l'état de ces dunes, l'ONF et le BRGM collaborent pour estimer l'intérêt des données de photogrammétrie acquises par drone pour l'évaluation de l'érosion éolienne.

Ce travail présente les premiers développements méthodologiques qui sont menés sur un site test du littoral des Pays de Monts en Vendée (85). La plage et l'arrière-plage de la Parée Grollier a fait l'objet de 2 acquisitions photogrammétriques diachroniques $(2015,2016)$ sur un linéaire de $1 \mathrm{~km}$ et une largeur de $600 \mathrm{~m}$. Les ortho-photographies générées ont une résolution de $3 \mathrm{~cm}$ tandis que le nuage de point $3 \mathrm{D}$ a été généré avec des résolutions de $5 \mathrm{~cm}$. Plusieurs types d'analyses sont pratiqués sur les données de nuage de points, les orthophotographies et les Modèles Numériques de Surface acquis par drone pour étudier les zones dévégétalisées et quantifier les processus d'érosion éolienne. Ces tests méthodologiques permettent de dégager les potentialités de ces nouvelles données pour analyser des états dunaires diversifiés (anthropisation, dégradation dunaire, végétalisation, etc.) et mieux caractériser l'évolution éolienne des dunes.

Mots-clés :

Dune littorale, Erosion éolienne, Drone, Photogrammétrie, Méthodologie. 


\section{Thème 3 - Instrumentation, mesures, imagerie et télédétection}

\section{Introduction}

L'utilisation des drones pour la surveillance des milieux naturels et le suivi des évolutions géomorphologiques permet d'atteindre des échelles de précision centimétrique et d'emprise inédite. L'accessibilité des nouvelles données photogrammétriques acquises à l'aide de drones favorise l'analyse de structures ou de processus naturels qui restent encore mal connus. Ces techniques d'acquisition sont en train d'atteindre une certaine maturité technologique et permettent une acquisition de forts volumes de données de qualité pour un coût relativement réduit (COLOMINA \& MOLINA, 2014).

Les différents milieux littoraux sableux peuvent être suivis avec ces nouveaux vecteurs : plages sableuses méditerranéenne (BELON et al., 2015 ; CASELLA et al., 2016 ), adriatique (MANCINI et al., 2013) ou de la côte Atlantique (GONCALVES et al., 2015), zone de baie et de flèches littorales de la côte atlantique française (LONG et al., 2016, STEPHAN et al., 2018), plages de poche de Bretagne (DELACOURT et al., 2009) ou de Guyane (BRUNIER et al., 2016).

Sur la côte Atlantique, l'ONF gère de très vastes massifs dunaires domaniaux et est en charge de la surveillance de ce milieu sensible. Tous les 5 ans, un inventaire exhaustif de l'état des dunes est réalisé afin d'évaluer leur état ainsi que l'efficacité des travaux de restauration engagés.

Dans le cadre du projet EPHEDRA (Evaluation des PHénomènes d'Erosion éolienne par utilisation de DRone sur les dunes du littoral Atlantique), l'ONF et le BRGM collaborent afin de tester des méthodes de traitement des données acquises par drone dans le but de faciliter et d'optimiser les travaux d'expertises menés par les techniciens forestiers sur le terrain.

Ce travail présente les premiers développements méthodologiques qui sont menés sur le domaine dunaire d'un site test du littoral des Pays de Monts en Vendée (85). Ce littoral sableux fait l'objet de suivis réguliers par l'Observatoire du Littoral des Pays de Monts depuis 2010 (LE GUERN et al., 2013). Il s'étend du goulet de Fromentine jusqu'à Notre-Dame-de-Monts (figure 1). Des dunes littorales y sont bien établies avec un front dunaire majoritairement en phase de régénération depuis la tempête Xynthia (2010). Ainsi, selon les données Lidar exploitées par LE MAUFF et al. (2018), les volumes érodés entre 2008 et 2010 ont été regagnés entre 2010 et 2013 par la construction d'une dune embryonnaire devant la cicatrice d'érosion induite par la tempête Xynthia. Le site d'étude est situé au nord de Notre-Dame-de-Monts, sur la plage de la Parée Grollier. Cette plage naturelle est éloignée de toute artificialisation, mais la dune est altérée par un important réseau de cheminements piétonniers 


\section{XVèmes Journées Nationales Génie Côtier - Génie Civil \\ La Rochelle, 29 au 31 mai 2018}
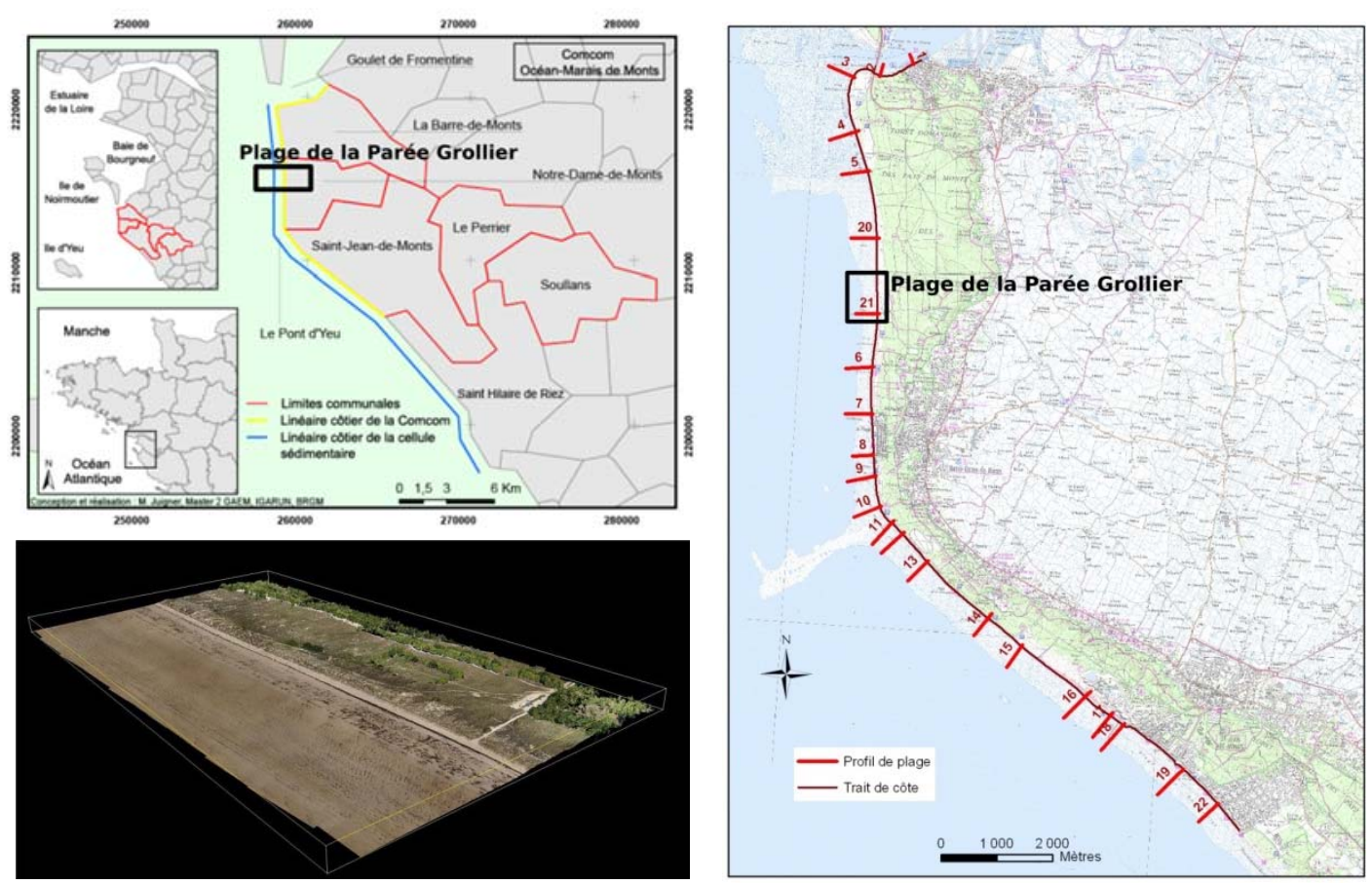

Figure 1. Localisation de la zone d'étude de l'OLPM, de la plage de la Parée Grollier et de la zone suivie par drone.

\section{Matériel et méthode}

\subsection{Campagne de mesure}

La plage et l'arrière-plage de la Parée Grollier a fait l'objet de deux acquisitions photogrammétriques diachroniques (octobre 2015 et octobre 2016) sur un linéaire de $1 \mathrm{~km}$ et une largeur de $600 \mathrm{~m}$, figure 1 . Les orthophotographies générées ont une résolution de $3 \mathrm{~cm}$ tandis que le nuage de point $3 \mathrm{D}$ a été généré avec des résolutions de $5 \mathrm{~cm}$. Le vol, la captation et le post-traitement des images brutes ont été réalisés par la société Azur Drone qui a mis en œuvre un modèle de drone à aile fixe de type AVEM conçu par la société Aeromapper et équipé d'un appareil photo numérique Sony A5100 de 24 Méga pixel. Ce type de drone présente l'avantage d'une bonne stabilité dans les airs et d'une grande autonomie en vol. Les vols ont été réalisés à marée basse de viveeau d'équinoxe (coefficients de marée de 108 en 2015 et 114 en 2016).

\subsection{Post-traitement des données brutes}

Le post traitement des images est réalisé à l'aide du logiciel Agisoft Photoscan. Un ensemble de 16 cibles positionnées au GPS centimétrique $(2 \mathrm{~cm}$ de précision) et réparties sur la plage et la dune ont permis le calage géographique des données. L'erreur moyenne de positionnement des cibles dans le modèle de photogrammétrie est de $4 \mathrm{~cm}$ en 2015 et $6 \mathrm{~cm}$ en 2016. Lors des mesures, la plage de la Parée Grollier présentait une 


\section{Thème 3 - Instrumentation, mesures, imagerie et télédétection}

surface suffisamment variée et texturée pour permettre au logiciel de procéder à la phase d'appariement des images nécessaire au calcul de géométrie. Cette variabilité des couleurs et des textures de la plage s'explique par la présence d'algues et de figures sédimentaires sur l'estran et par la morphologie et la végétation contrastée de la dune. Cependant, pour certains secteurs de la plage, l'algorithme du logiciel n'a pas permis de détecter suffisamment de points communs entre images pour permettre l'alignement des images et un calcul optimal de la géométrie. Des tests réalisés ont montré que les traces de pas et déplacements sur l'estran apparus pendant la durée du levé ont altéré localement la qualité du positionnement. Pour les deux levés (octobre 2015 et octobre 2016), cette altération locale se traduit par des secteurs présentant des données d'altitude ponctuellement bruitées : dans un secteur de $20 \mathrm{~cm}$ autour de chaque point, le bruit moyen des zones "précises" est de $0,5 \mathrm{~cm}$ (maximum $1 \mathrm{~cm}$ ) alors qu'il est de $1 \mathrm{~cm}$ (maximum $4 \mathrm{~cm}$ ) dans les zones "bruitées". Au bilan, la précision moyenne des mesures d'altitudes est estimée à $8 \mathrm{~cm}$ pour 2015 et $10 \mathrm{~cm}$ en 2016 pour des cellules de $20 \mathrm{~cm}$.

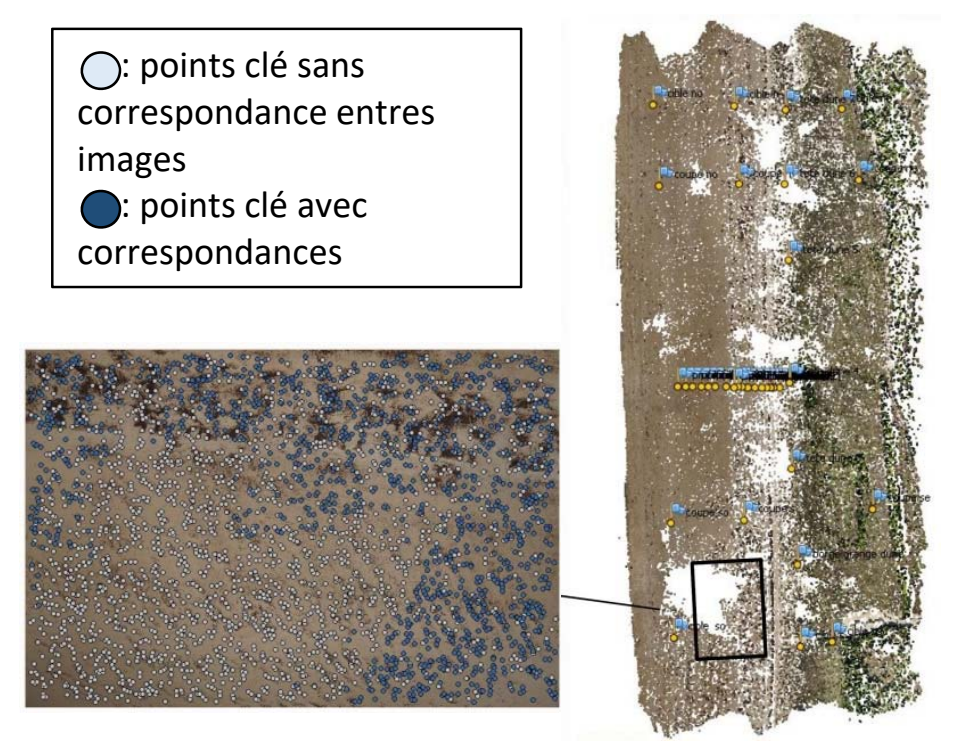

Figure 2. Résultats du post-traitement des images brutes.

\subsection{Méthode d'analyse des données}

La méthode d'analyse proposée se base sur l'utilisation des deux types d'informations produites à partir des images acquises par drone.

Dans un premier temps, les ortho-photographies ont été exploitées pour extraire les zones dunaires sans végétation. L'agrégation et le seuillage adapté à la prise de vue a permis une première sélection rapide des principaux secteurs sans végétation. Un travail de validation-correction de cette sélection automatique a permis ensuite d'affiner la précision de la cartographie des zones non-végétalisées à l'aide d'un SIG. La très fine résolution des données utilisée favorise l'extraction de zones de cheminement très 


\section{XVèmes Journées Nationales Génie Côtier - Génie Civil \\ La Rochelle, 29 au 31 mai 2018}

étroites (inférieure à $50 \mathrm{~cm}$ de large) située sur la dune mobile dite "blanche" ou de petits secteurs situés dans la dune fixée dite "grise" et affectés très localement.

Dans un second temps, les nuages de points ont été utilisés pour produire des modèles numériques de surfaces (MNS) aisément exploitables sous SIG. Ces modèles représentent la position tridimensionnelle de chaque pixel apparié. Dans les zones densément végétalisées, le MNS fourni l'altitude du sommet de la végétation et non l'altitude du sol, qui n'est pas détecté avec ces techniques photogrammétriques. Les différentiels d'altitude sont donc extraits des surfaces non végétalisées pour estimer si des effets d'érosion ou d'accrétion ont eu lieu entre les deux campagnes. Un travail de développement méthodologique basé plus directement sur les nuages des points est en cours afin d'améliorer la précision des résultats (figure 3).

\section{Résultats}

\subsection{Cartographie des surfaces dunaires non végétalisées}

Pour le secteur d'étude de la Parée Grollier, les zones dunaires non végétalisées représentent $18 \%$ de la surface suivie par drone, soit 3,6 ha non végétalisés sur 20,5 ha de zone dunaire non boisée délimitée entre la clôture du pied de dune et le chemin bordant la forêt. La figure 3 met en évidence les secteurs non-végétalisés détectés sur l'orthophotographie drone par reconnaissance semi-automatique.
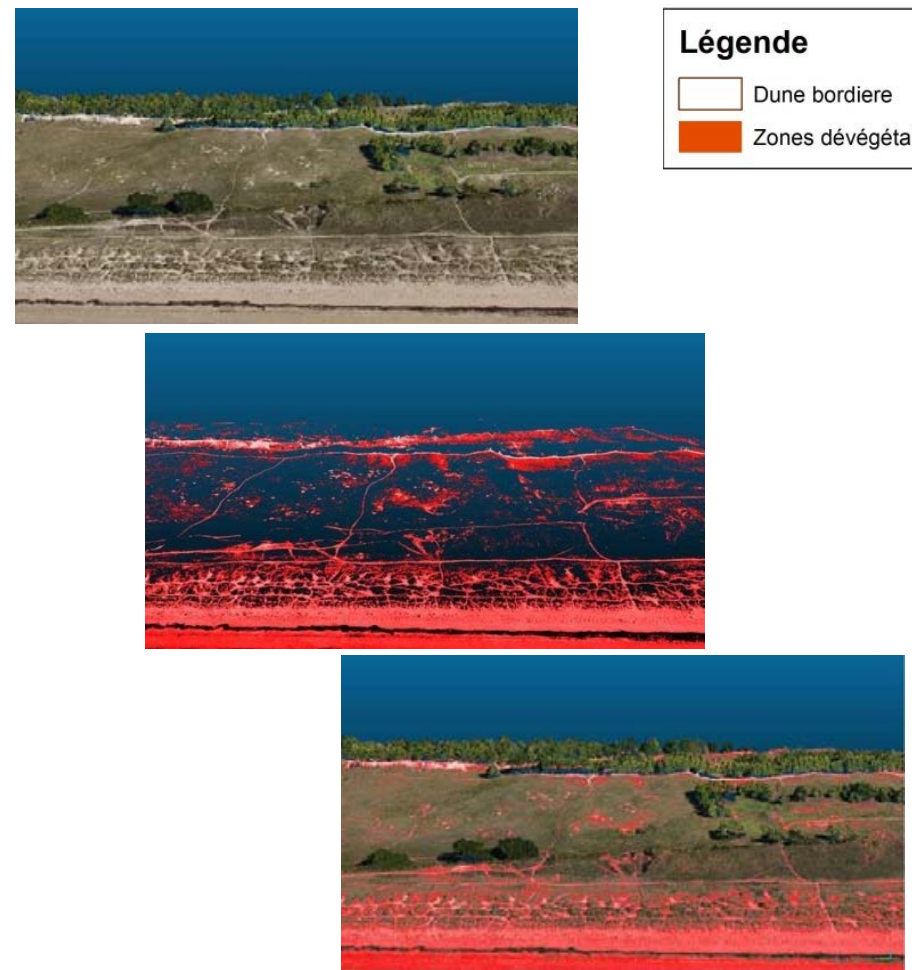
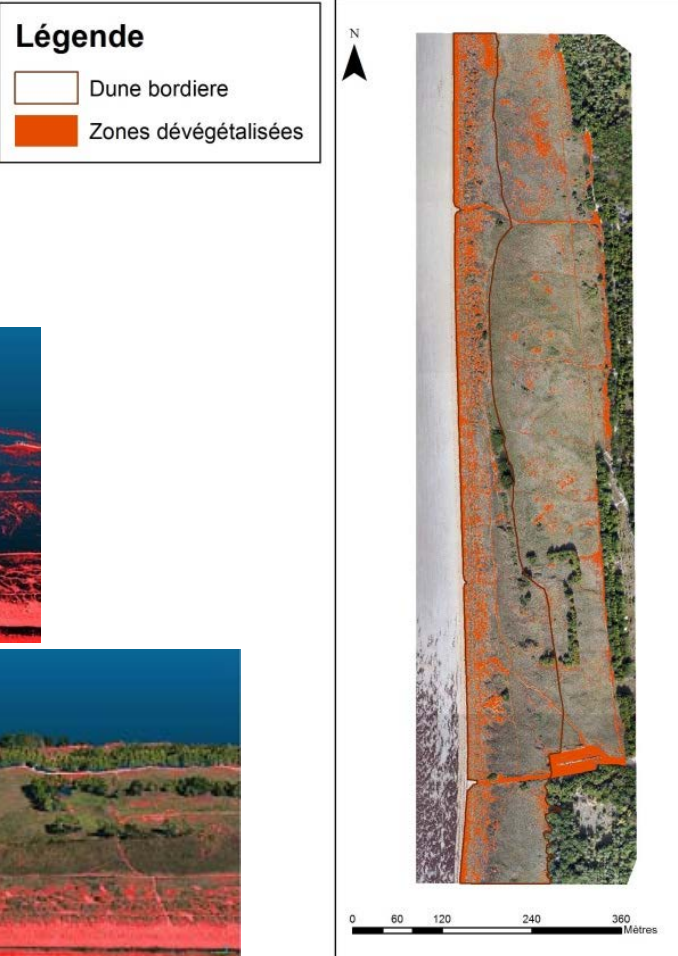

Figure 3. Détection semi-automatique des zones non-vegétalisées depuis le nuage de points et les ortho-photographies. 


\section{Thème 3 - Instrumentation, mesures, imagerie et télédétection}

Le pied de dune représente $2 \%$ de la zone non végétalisée. La dune bordière couvre une surface de 9 ha sur la zone d'étude (44,5\% de la surface étudiée). Les zones qui y sont dégradées correspondent à $59,5 \%$ du total des surfaces non-végétalisées, le reste (40,5\%) étant situé en arrière de la dune, au niveau de la zone basse (la lette) située entre la dune et la forêt.

L'absence de végétation est expliquée par plusieurs facteurs :

- l'artificialisation au niveau du parking et du cheminement principal d'accès à la plage ;

- les sentiers "canalisés" par des clôtures, au sommet de dune et au pied du talus ;

- les zones de cheminement non canalisés, denses, et situées sur le front dunaire ;

- plusieurs zones de déflation éolienne, de faibles emprises et localisées au niveau du front dunaire, ou plus vaste à l'arrière de la dune.

- la présence de terriers dans les pelouses de la lette qui forment des ensembles de tâches sableuses.

\subsection{Evolutions morphologiques}

Les nuages de points issus du traitement photogrammétrique ont permis la réalisation de MNS de $30 \mathrm{~cm}$ de résolution au sol en 2015 et 2016. Le différentiel d'altitude établi entre ces deux dates montre des évolutions morphologiques significatives traduisant des phénomènes d'érosion éolienne par déflation et une dégradation du couvert végétal par endroits. A cette échelle d'étude et considérant la précision moyenne des données brutes, nous considérons que des évolutions supérieures à $15 \mathrm{~cm}$ sur des surfaces de plusieurs cellules de $30 \mathrm{~cm}$ peuvent être considérées comme significatives. Ainsi, la figure 4 (a) montre plusieurs petits secteurs de cheminement qui se sont érodés de 15 à $20 \mathrm{~cm}$ entre 2015 et 2016 à cause d'un piétinement important. Sur des surfaces dégradées plurimétriques (b), les différences d'altitude peuvent atteindre 30 à $50 \mathrm{~cm}$ très localement car les secteurs plus larges deviennent très sensibles à l'érosion éolienne. Le différentiel topographique montre également des tendances locales à l'exhaussement, comme au niveau du pied de dune (c) qui est un secteur en accrétion régulière grâce aux apports du sable de plage et sur lequel la végétation se développe rapidement.

Au niveau de la dune blanche, où la végétation est suffisamment haute, l'association du différentiel topographique et des ortho-photographies permet de détecter des secteurs de rapide dégradation de la végétation. Ainsi, la figure 5 montre un point très localisé présentant un différentiel négatif observé de 50 à $80 \mathrm{~cm}$ entre 2015 et 2016 . Une telle différence d'altitude est liée à la fois à l'arrachage artificiel de la végétation (dégradations volontaires par des touristes occupant les dunes) et à un surcreusement de la dune. 


\section{XVèmes Journées Nationales Génie Côtier - Génie Civil La Rochelle, 29 au 31 mai 2018}

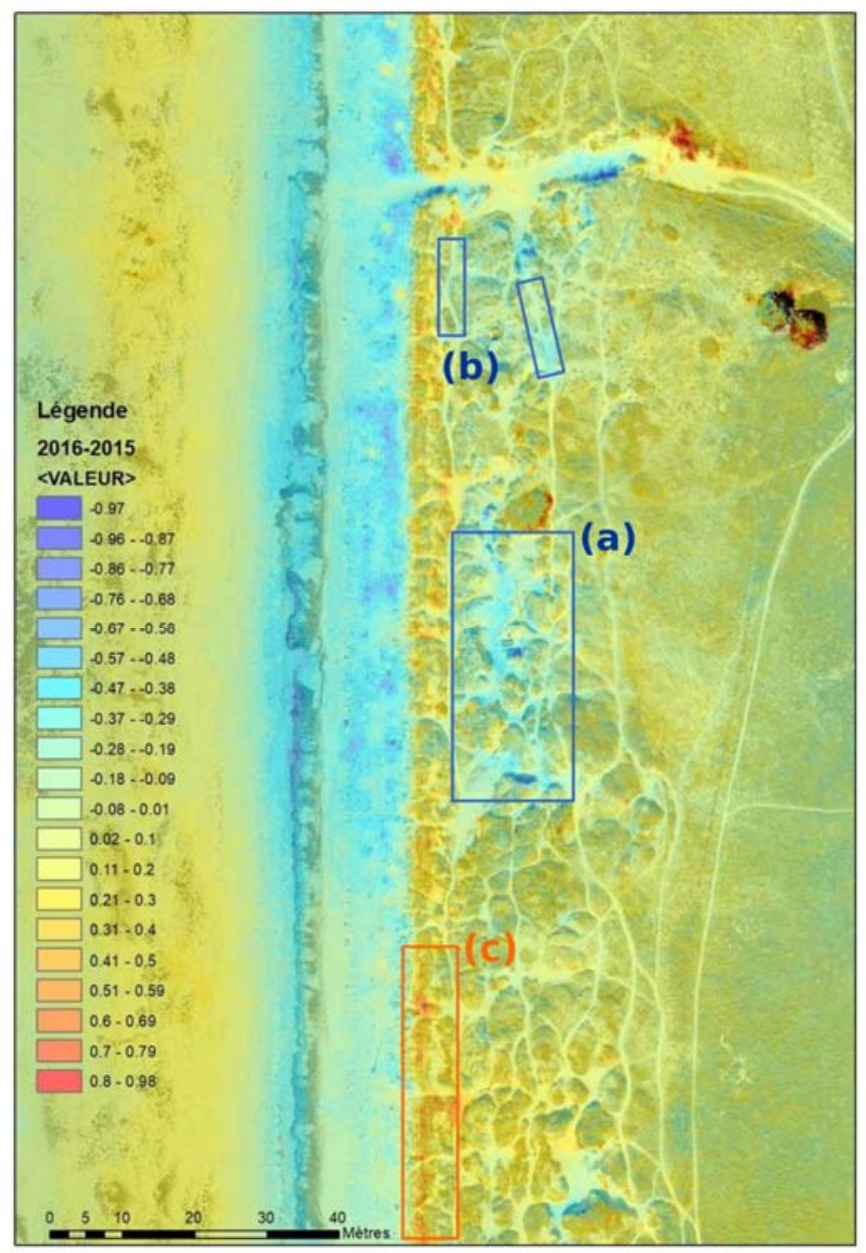

Figure 4. Extrait du différentiel 2015-2016 entre les MNS issus des levés drones.
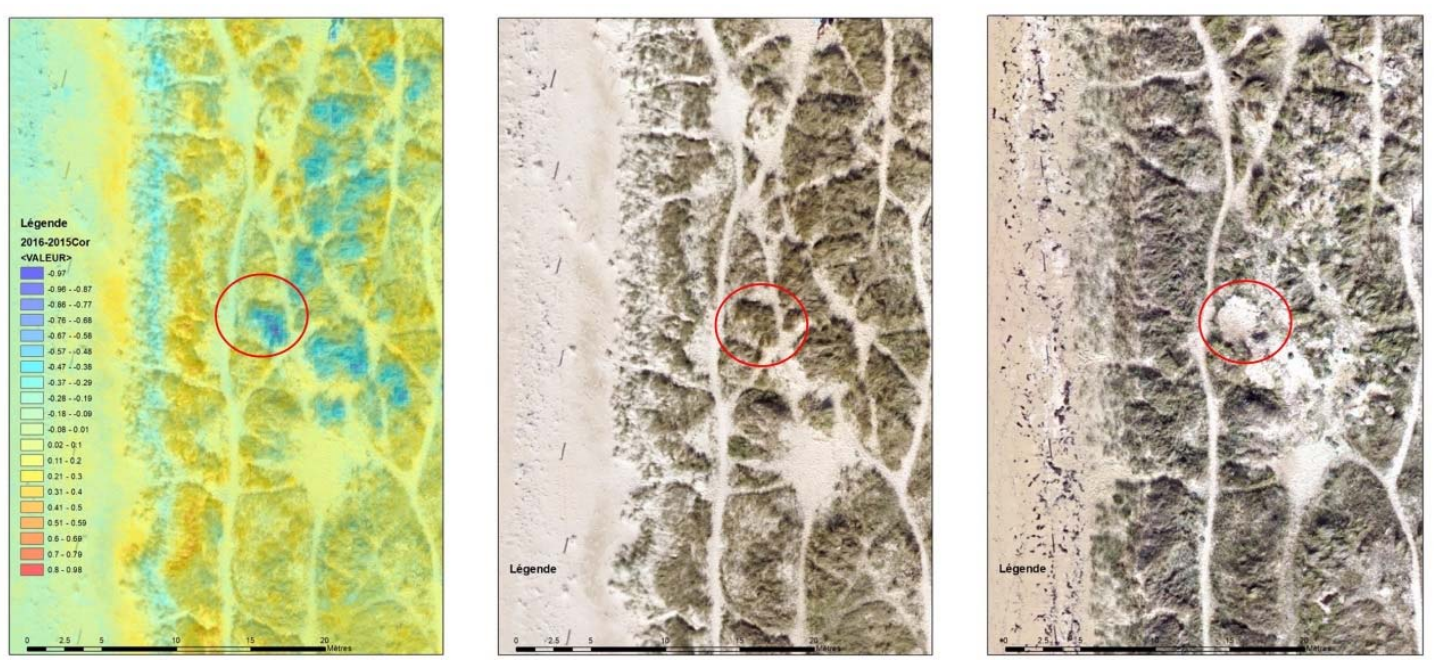

Figure 5. Utilisation du différentiel d'altitude 2015-2016 et des ortho-photographies pour localiser une zone de dégradation locale de la végétation dunaire et d'érosion. 


\section{Thème 3 - Instrumentation, mesures, imagerie et télédétection}

\section{Conclusions}

La plage de la Parée Grollier (Notre-Dame-de-Monts, Vendée) a été suivie en 2015 et 2016 par deux levés photogrammétriques par drone dans le cadre de travaux méthodologiques du BRGM. Le front dunaire apparait comme fortement dégradé par la surfréquentation et le piétinement en dehors des sentiers balisés. La dune blanche y est sujette à la déflation éolienne sur les secteurs non-végétalisés. La plage de la Parée Grollier apparait donc comme un premier site-atelier adapté aux besoins du projet EPHEDRA, il sera complété par un second site de suivi complémentaire en 2018.

Les tests méthodologiques réalisés sur les données photogrammétriques ont permis de cartographier finement les zones non-végétalisées situées entre le pied de dune et la zone boisée et de quantifier la dégradation du couvert végétal du massif dunaire entre octobre 2015 et octobre 2016. La très grande résolution des données favorise la détection de très petites surfaces soumises à l'érosion éolienne au sein des morphologies complexes de la dune. Le couplage des analyses d'orthophotographie et de MNS (ou directement des données des nuages de points) offre des perspectives nouvelles pour identifier précisément et comprendre la nature des modifications de couverture végétale et de morphologie dans les zones dunaires.

\section{Références bibliographiques}

BELON R., DE LA TORRE Y., SIBERT V., MOUROU X. (2015). Contribution des relevés photogrammétriques par drone pour l'analyse de la dynamique côtière. Application sur un site de la côte sableuse catalane. 3ème Conférence Méditerranéenne Côtière et Maritime, Ferrara, Italia, Ed. Paralia, pp 1-6. https://doi.org/10.5150/cmcm.2015.001 BRUNIER G., FLEURY J., ANTHONY E. J., GARDEL A., DUSSOUILLEZ P. (2016). Close-range airborne Structure-from-Motion Photogrammetry for highresolution beach morphometric surveys: Examples from an embayed rotating beach. Geomorphology, Vol. 261, pp 76-88. https://doi.org/10.4000/geomorphologie.11358

CASElla E., ROVERE A., PEDRONCINI A., STARK C.P., CASELlA M., FERRARI M., FIRPO M. (2016). Drones as tools for monitoring beach topography changes in the Ligurian Sea (NW Mediterranean). Geo-Marine Letters, Vol. 36(2), pp 151-163. https://doi.org/10.1007/s00367-016-0435-9

COLOMINA I., MOLINA P. (2014). Unmanned aerial systems for photogrammetry and remote sensing: A review. ISPRS Journal of Photogrammetry and Remote Sensing, Vol. 92, pp 79-97. https://doi.org/10.1016/j.isprsjprs.2014.02.013

DELACOURT C., ALLEMAND P., JAUD. M., GRANDJEAN P., DESCHAMPS A., AMMANN J., CUQ V., SUANEZ S. (2009). DRELIO: An Unmanned Helicopter for Imaging Coastal Areas. Journal of Coastal Research, ICS2009 (Proceedings), (SI 56), pp 1489-1493. 


\section{XVèmes Journées Nationales Génie Côtier - Génie Civil \\ La Rochelle, 29 au 31 mai 2018}

GONCALVES J.A., HENRIQUES R. (2015). UAV photogrammetry for topographic monitoring of coastal areas. ISPRS Journal of Photogrammetry and Remote Sensing, Vol. 104, pp 101-111. https://doi.org/10.1016/j.isprsiprs.2015.02.009

LE MAUFF B., JUIGNER M., BA A., ROBIN M., LAUNEAU P., FATTAL P. (2018). Coastal monitoring solutions of the geomorphological response of beach-dune systems using multi-temporal LiDAR datasets (Vendée coast, France). Geomorphology, Vol. 304, pp 121-140. https://doi.org/10.1016/j.geomorph.2017.12.037

LE GUERN C., JUIGNIER M., BAUDOUIN V., ROBIN M., FATTAL P., ROLLIER C., MARIE D., MAANAN M. (2013). Observatoire du littoral des Pays de Monts Synthèse 2012 des réalisations des partenaires scientifiques (IGARUN, ONF, BRGM), Rapport d'avancement 2012. BRGM RP-62937-FR, 95 p.

LONG N., MILLESCAMPS B., GUILLOT B., POUGET F., BERTIN X. (2016). Monitoring the Topography of a Dynamic Tidal Inlet Using UAV Imagery. Remote Sensing, Vol. 8(387), 18 p. https://doi.org/10.3390/rs8050387

MANCINI F., DUBBINI M., GATTELLI M., STECCHI F., FABBRI F., GABBIANELLI G. (2013). Using Unmanned Aerial Vehicles (UAV) for HighResolution Reconstruction of Topography: The Structure from Motion Approach on Coastal Environments. Remote Sensing, Vol. 5, pp 6880-6898. https://doi.org/10.3390/rs5126880

STEPHAN P., SUANEZ S., FICHAUT B., AUTRET R., BLAISE E., HOURON J., AMMANN J., GRANJEAN P. (2018). Monitoring the medium-term retreat of a gravel spit barrier and management strategies, Sillon de Talbert (North Brittany, France). Ocean \& Coastal Management, Vol. 158, pp 64-82. 
Thème 3 - Instrumentation, mesures, imagerie et télédétection 